DESY 05-166

\title{
EXTREMELY ENERGETIC COSMIC NEUTRINOS: OPPORTUNITIES FOR ASTROPHYSICS, PARTICLE PHYSICS, AND COSMOLOGY *
}

\author{
ANDREAS RINGWALD \\ Deutsches Elektronen-Synchrotron DESY, \\ Notkestraße 85, \\ D-22607 Hamburg, Germany \\ E-mail: andreas.ringwald@desy.de
}

\begin{abstract}
Existing and planned observatories for cosmic neutrinos open up a huge window in energy from $10^{7}$ to $10^{17} \mathrm{GeV}$. Here, we discuss in particular the possibilities to use extremely energetic cosmic neutrinos as a diagnostic of astrophysical processes, as a tool for particle physics beyond the Standard Model, and as a probe of cosmology.
\end{abstract}

\section{Introduction}

We are living in exciting times for extremely high energy cosmic neutrinos (EHEC $\nu$ 's). Existing observatories, such as AMANDA ${ }^{1}$, ANITA-lite ${ }^{2}$, $\mathrm{BAIKAL}^{3}, \mathrm{FORTE}^{4}, \mathrm{GLUE}^{5}$, and $\mathrm{RICE}^{6}$ have recently put restrictive upper limits on the neutrino flux in the energy region from $10^{7}$ to $10^{17} \mathrm{GeV}$ (cf. Fig. 1). Furthermore, recent proposals for larger EHEC $\nu$ detectors, such as ANITA ${ }^{7}, \mathrm{EUSO}^{8}$, IceCube ${ }^{9}, \mathrm{LOFAR}^{10}, \mathrm{OWL}^{11}, \mathrm{PAO}^{12}, \mathrm{SalSA}^{13}$, $\mathrm{WSRT}^{10}$, together with conservative neutrino flux predictions from astrophysical sources of the observed cosmic rays (CR's), such as active galactic nuclei, offer credible hope that the collection of a huge event sample above $10^{7} \mathrm{GeV}$ may be realized within this decade (cf. Fig. 1). This will provide not only important information on the astrophysical processes associated with the acceleration of CR's, but also an opportunity for particle physics beyond the reach of the Large Hadron Collider (LHC). There is even the possibility of a sizeable event sample above $10^{11} \mathrm{GeV}$, with important consequences for cosmology. The corresponding neutrino fluxes may arise from the decomposition of topological defects - relics of phase transitions in the very early universe - into their particle constituents. Moreover, it may be possible to detect the cosmic neutrino background via absorption features in these neutrino spectra. In this contribution, we will have a closer look at these exciting opportunities.

*Talk presented at the ARENA Workshop, DESY, Zeuthen, Germany, May 17-19, 2005. 


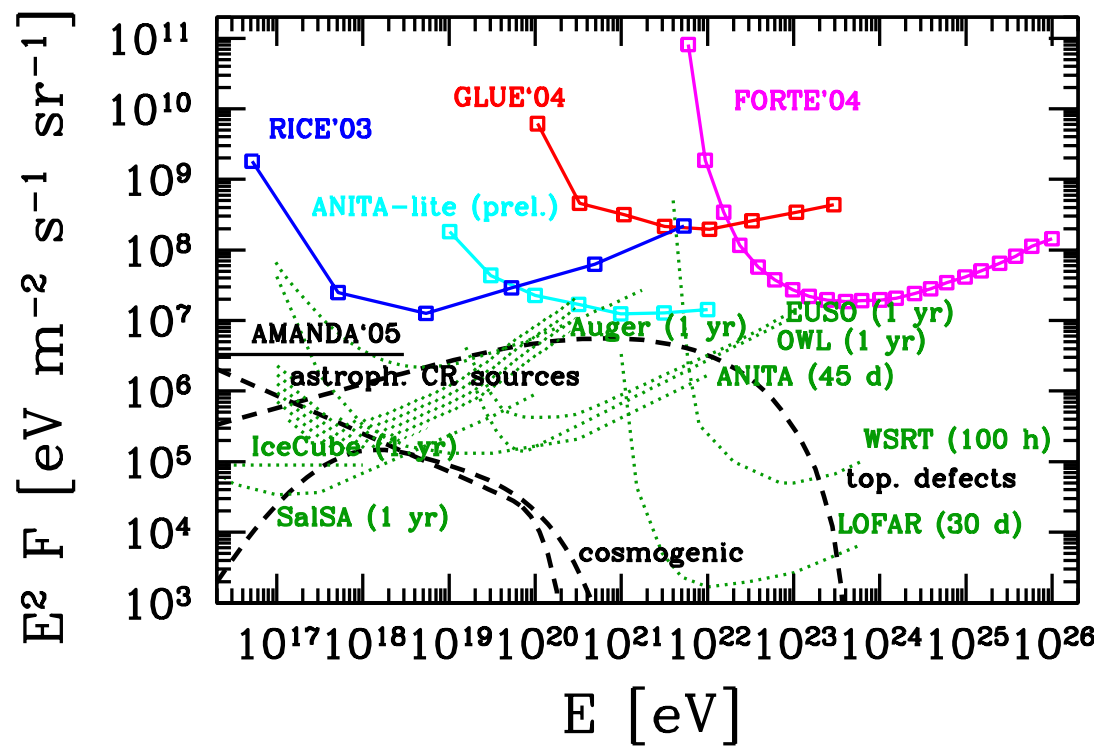

Figure 1. Current status and next decade prospects for EHEC $\nu$ physics, expressed in terms of diffuse neutrino fluxes per flavor, $F_{\nu_{\alpha}}+F_{\bar{\nu}_{\alpha}}, \alpha=e, \mu, \tau$. Upper limits from AMANDA $^{1}$, ANITA-lite ${ }^{2}$, FORTE $^{4}, \mathrm{GLUE}^{5}$, and RICE ${ }^{6}$. Also shown are projected sensitivities of $\mathrm{ANITA}^{7}, \mathrm{EUSO}^{8}, \mathrm{IceCube}^{9}, \mathrm{LOFAR}^{10}, \mathrm{OWL}^{11}$, the Pierre Auger Observatory in $\nu_{e}, \nu_{\mu}$ modes and in $\nu_{\tau}$ mode (bottom swath) ${ }^{12}, \operatorname{SalSA}^{13}$, and $\mathrm{WSRT}^{10}$, corresponding to one event per energy decade and indicated duration. Also shown are predictions from astrophysical CR sources ${ }^{14}$, from inelastic interactions of CR's with the cosmic microwave background (CMB) photons (cosmogenic neutrinos $)^{14,15}$, and from topological defects ${ }^{16}$.

\section{EHEC $\nu$ 's as a diagnostic of astrophysical processes}

Neutrinos with energies $\lesssim 10^{12} \mathrm{GeV}$ propagate essentially without interaction between their source and Earth. Hence, they are a powerful probe of high energy astrophysics, in particular of the conjectured acceleration sites of the CR's, notably active galactic nuclei (AGN). A paradigm for the acceleration mechanism in the jets of these AGN's is shock acceleration. Protons and heavier nuclei are confined by magnetic fields and accelerated through repeated scattering by plasma shock fronts. Inelastic collisions of the trapped protons with the ambient plasma produces pions and neutrons, the former decaying into neutrinos and photons, the latter eventually diffusing from the source and decaying into CR protons (cf. Fig. 2 (left)).

A quite conservative estimate of the flux of neutrinos from such astro- 

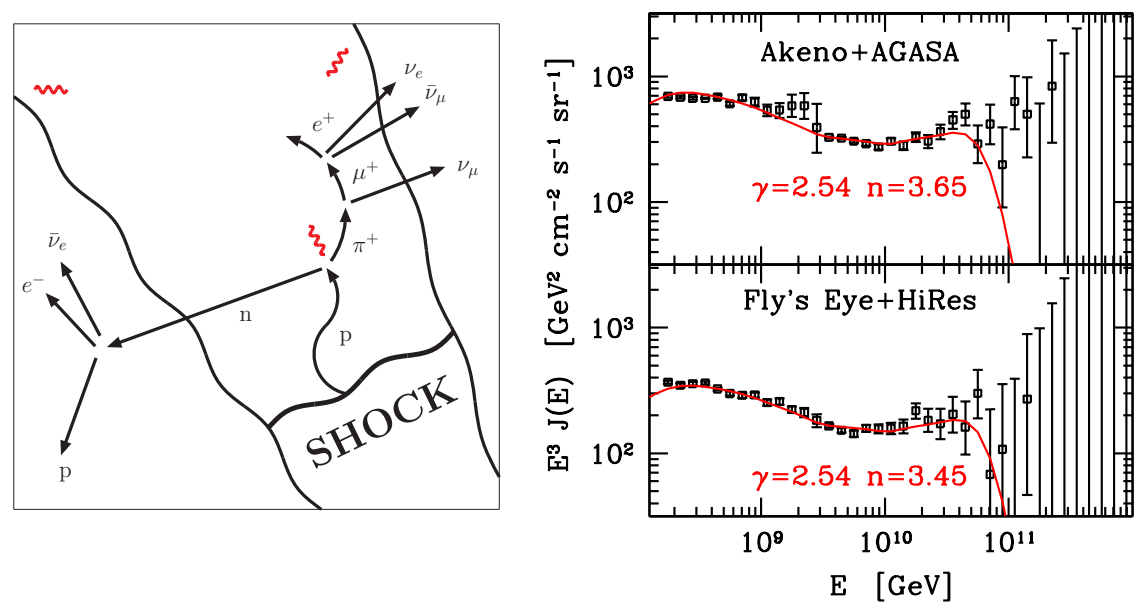

Figure 2. Left: Illustration of shock acceleration in the jet of an active galaxy ${ }^{14}$. Right: Best fits ${ }^{14}$ to the ultra-high energy cosmic ray spectrum in the energy interval $\left[10^{8.6}, 10^{11}\right] \mathrm{GeV}$ as observed by Akeno ${ }^{18}+$ AGASA $^{19}$ and Fly's Eye ${ }^{20}+$ HiRes $^{21}$. The dip from $e^{+} e^{-}$pair production ${ }^{22,23}$ and the bump from Greisen-Zatsepin-Kuzmin ${ }^{24}$ (GZK) accumulation are clearly visible in the data and support the simple power law ansatz for the emissivity of the extragalactic sources, in which we have set $z_{\min }=0.012$, $z_{\max }=2$, and $E_{i, \max }=10^{12.5} \mathrm{GeV}$, for the boundaries in redshift and injection energy, respectively. Apparently, this fit undershoots the data for the few highest energy events.

physical sources can be made as follows ${ }^{14}$. Assuming that the sources are optically thin, i.e. the neutrons can escape, one may determine the neutron emissivity at the sources from the observed CR spectra ${ }^{17}$, taking into account propagation effects, in particular $e^{+} e^{-}$and pion production through inelastic scattering off the CMB photons. Figure 2 (right) illustrates that both the AGASA and the HiRes data in the $10^{8.6 \div 11} \mathrm{GeV}$ range can be fitted nicely under the assumption of a simple power law neutron injection emissivity, $\propto E_{i}^{-2.5}(1+z)^{3.5}$, of the extragalactic sources, supporting the recent proposal towards a low transition energy, $\sim 10^{8.6} \mathrm{GeV}$, between galactic and extragalactic cosmic rays ${ }^{22}$, which is also sustained by chemical composition studies of HiRes data ${ }^{25}$. The neutron injection emissivity is simply related to the neutrino emissivity, and the latter can be translated easily into an expected neutrino flux at Earth. It should be detected very soon, if not already with AMANDA-II, then at least with IceCube (cf. Fig. 1), which therefore can provide significant clues in demarcating the cosmic ray galactic/extragalactic crossover energy ${ }^{14}$. Although the cosmogenic neutrino flux from the inelastic interactions with the CMB photons 

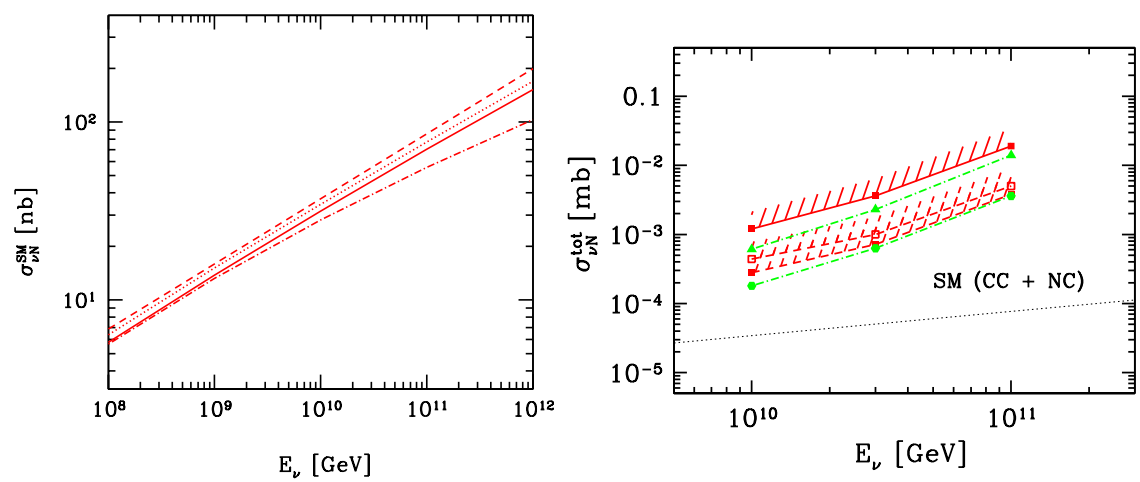

Figure 3. Left: Standard Model $\nu N$ total cross section $\sigma_{\nu N}^{\text {tot }}$ at extremely high neutrino energies $E_{\nu}$ obtained by various perturbative QCD resummation techniques ${ }^{26}$ : from a unified BFKL-DGLAP approach ${ }^{27}$ (solid), based on CTEQ parton distributions ${ }^{28}$ (dotted), based on GRV dynamical partons ${ }^{29}$ (dashed), and from a unified BFKLDGLAP approach supplemented by saturation effects ${ }^{30}$ (dashed-dotted). Right: Modelindependent upper bounds on the neutrino-nucleon inelastic cross section ${ }^{40}$ derived from the RICE Collaboration search results ${ }^{6}$, by exploiting different cosmogenic neutrino flux estimates, by Fodor et al. (FKRT $\left.{ }^{15}\right)$ (solid line) and Protheroe and Johnson $\left(\mathrm{PJ}^{39}\right.$ ) (dashed line joining solid squares). The dashed line joining the open squares (PJ) indicates the upper bound for inelasticity $\langle y\rangle=0.5$. The dashed-dotted lines indicate the sensitivity $\left(95 \% \mathrm{CL}\right.$, for $\sigma_{\nu N}^{\text {tot }}<4 \mathrm{mb}$ ) of PAO in $10 \mathrm{yr}$ of operation assuming zero events observed above SM background (circles PJ, triangles FKRT). For comparison, also shown is the SM total (charged current and neutral current) $\nu N$ inelastic cross section ${ }^{28}$.

starts to dominate over the neutrino flux from optically thin cosmic ray sources at energies above a few $\mathrm{EeV}$, it appears to be hard to detect with the EHEC $\nu$ detectors operating in the next decade (cf. Fig. 1).

\section{EHEC $\nu$ 's and physics beyond the Standard Model}

Cosmic neutrinos with energies $E_{\nu}$ above $10^{8} \mathrm{GeV}$ probe neutrino-nucleon scattering at center-of-mass (c.m.) energies above

$$
\sqrt{s_{\nu N}} \equiv \sqrt{2 m_{N} E_{\nu}} \simeq 14 \mathrm{TeV}\left(E_{\nu} / 10^{8} \mathrm{GeV}\right)^{1 / 2},
$$

beyond the proton-proton c.m. energy $\sqrt{s_{p p}}=14 \mathrm{TeV}$ of the LHC, and Bjorken $x \equiv Q^{2} /\left(y s_{\nu N}\right)$ values below

$$
x \simeq 2 \times 10^{-4}\left(Q^{2} / m_{W}^{2}\right)(0.2 / y)\left(10^{8} \mathrm{GeV} / E_{\nu}\right),
$$

where $Q^{2}$ is the momentum transfer squared, $m_{W} \simeq 80 \mathrm{GeV}$ the $W$-boson mass, and $y$ the inelasticity parameter. Under these kinematical conditions, the predictions for $\nu N$ scattering from the perturbative Standard Model 
(SM) are quite safely under control (cf. Fig. 3 (left)), notably thanks to the input from measurements of deep-inelastic ep scattering at HERA ${ }^{31,32}$. This makes it possible to search for enhancements in the $\nu N$ cross section due to physics beyond the (perturbative) SM, such as electroweak sphaleron $^{33}$ (non-perturbative $B+L$ violation), or Kaluza-Klein, black hole, $p$-brane, or string ball production in TeV scale gravity models ${ }^{34}$.

Since the rate of neutrino-initiated showers is proportional to integrated flux times cross section, the non-observation of quasi-horizontal or deeplypenetrating neutrino-induced air showers as reported by, e.g., Fly's Eye $\mathrm{E}^{35}$, $\mathrm{AGASA}^{36}$, and $\mathrm{RICE}^{6}$ can be turned into an upper bound on the neutrino nucleon cross section if a certain prediction for the neutrino flux is exploited $^{37,38}$. This is exemplified in Fig. 3 (right), which displays the limits on $\sigma_{\nu N}$ from the RICE search results on contained showers ${ }^{40}$, for two different assumptions about the $\mathrm{EHEC} \nu$ flux. These bounds are considerably higher than the SM cross section, albeit in the post-LHC energy region. $\mathrm{PAO}$ will be able to improve these limits by one order of magnitude ${ }^{40}$.

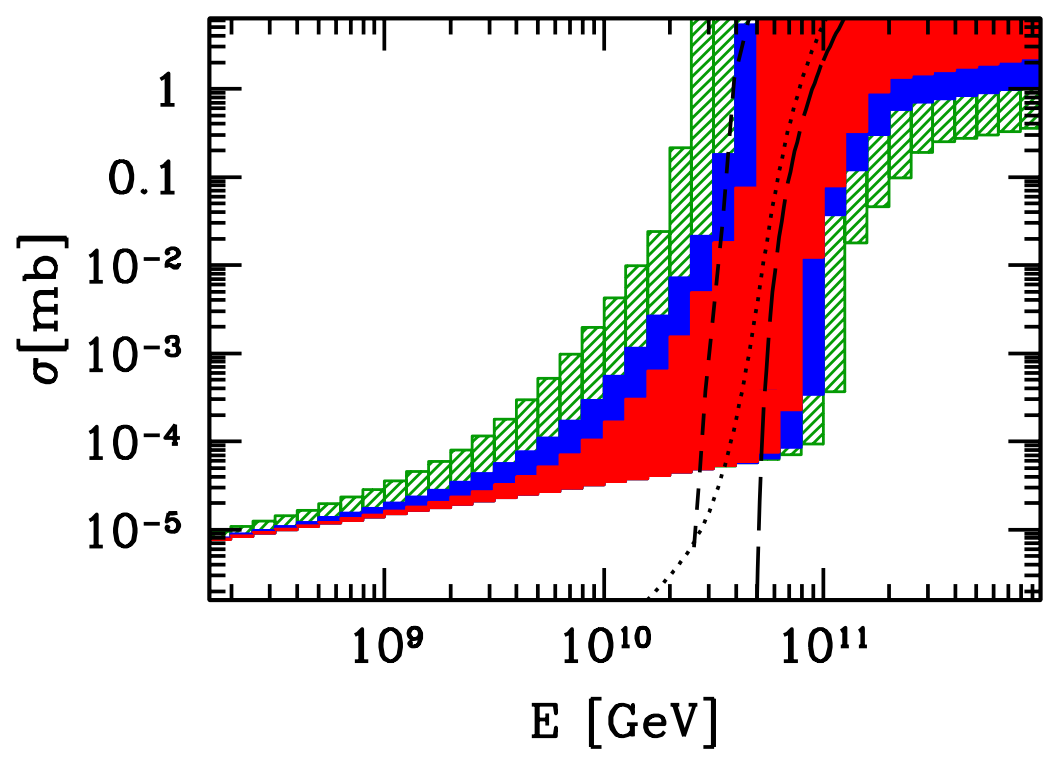

Figure 4. The range of the cross section within the 99\%, 95\% and 90\% CL required for a successful strongly interacting neutrino scenario ${ }^{42}$. The lines are theoretical predictions of an enhancement of the neutrino-nucleon cross-section by electroweak sphalerons ${ }^{43,44}$ (short-dashed), $p$-branes ${ }^{45}$ (long-dashed) and string excitations ${ }^{46}$ (dotted). 
The bounds exploiting searches for deeply-penetrating particles are typically applicable as long as $\sigma_{\nu N} \lesssim 0.5 \div 1 \mathrm{mb}$. Models with even higher and more speculative cross sections, $\gtrsim 1 \div 10 \mathrm{mb}$, such as electroweak sphaleron production, brane production, or string resonance production, qualify as strongly interacting neutrino scenarios ${ }^{41,23}$, according to which the mysterious EHECR beyond the predicted GZK cutoff ${ }^{24}$ at $E_{\mathrm{GZK}} \simeq 4 \times 10^{10} \mathrm{GeV}$ (cf. Fig. 2 (right)) are initiated by cosmogenic neutrinos. Figure 4 illustrates that a combined fit of the existent data on vertical showers by AGASA and HiRes, as well as of the search results on weakly interacting particles of AGASA and RICE, requires a steep increase within one energy decade around $E_{\mathrm{GZK}}$ by four orders of magnitude ${ }^{42}$ - an enhancement which has indeed been proposed within some extensions of the (perturbative) SM.

We have emphasized here the current constraints from EHEC $\nu$ on physics beyond the SM. A more detailed account of the particle physics reach of the planned $\mathrm{EHEC} \nu$ observatories can be found elsewhere ${ }^{47,48}$.

\section{EHEC $\nu$ 's as a tool to study big bang relics}

The existence of topological stable solutions of the field equations (topological defects) is a generic prediction of symmetry breaking (SB) in Grand Unified Theories (GUT's) and occurs even at the fundamental level in String Theory in the form of F- and D-strings ${ }^{49}$. Specifically, $\mathrm{G} \rightarrow \mathrm{H} \times \mathrm{U}(1)$ SB leads to monopoles, U(1) SB to ordinary or superconducting strings, and $\mathrm{G} \rightarrow \mathrm{H} \times \mathrm{U}(1) \rightarrow \mathrm{H} \times \mathrm{Z}_{N}$ SB to monopoles connected by strings, e.g. necklaces in case of $N=2$. Such topological defects may be produced through non-thermal phase transitions during preheating after inflation ${ }^{50}$. Their superheavy constituents $X$, often gauge or Higgs bosons with masses $m_{X} \sim 10^{12 \div 16} \mathrm{GeV}$, may be liberated on various occasions ${ }^{51}$, e.g. through repeated self-intersections of strings, through annihilation of monopole antimonopole pairs etc., and rapidly decay into stable SM particles, under which we readily find ${ }^{52} \mathrm{EHEC} \nu$ 's with energies up to $\sim 0.05 m_{X}$. The corresponding fragmentation spectra are meanwhile worked out very accurately ${ }^{53}$ via Monte Carlo generators ${ }^{54}$ or via DGLAP evolution ${ }^{55}$ from experimentally determined initial distributions at the scale $m_{Z}$ to the ones at $m_{X}$. The injection rate, which determines in particular the overall normalization of the neutrino flux, depends on cosmic time $t$ in the form $\dot{n}_{X}=\kappa m_{X}^{p} t^{-4+p}$, where $\kappa$ and $p$ are dimensionless constants depending on the specific scenario ${ }^{52}$.

For a wide range of overall flux normalizations, the upcoming EHEC $\nu$ observatories seem to be sensitive enough to obtain, within the next decade, 


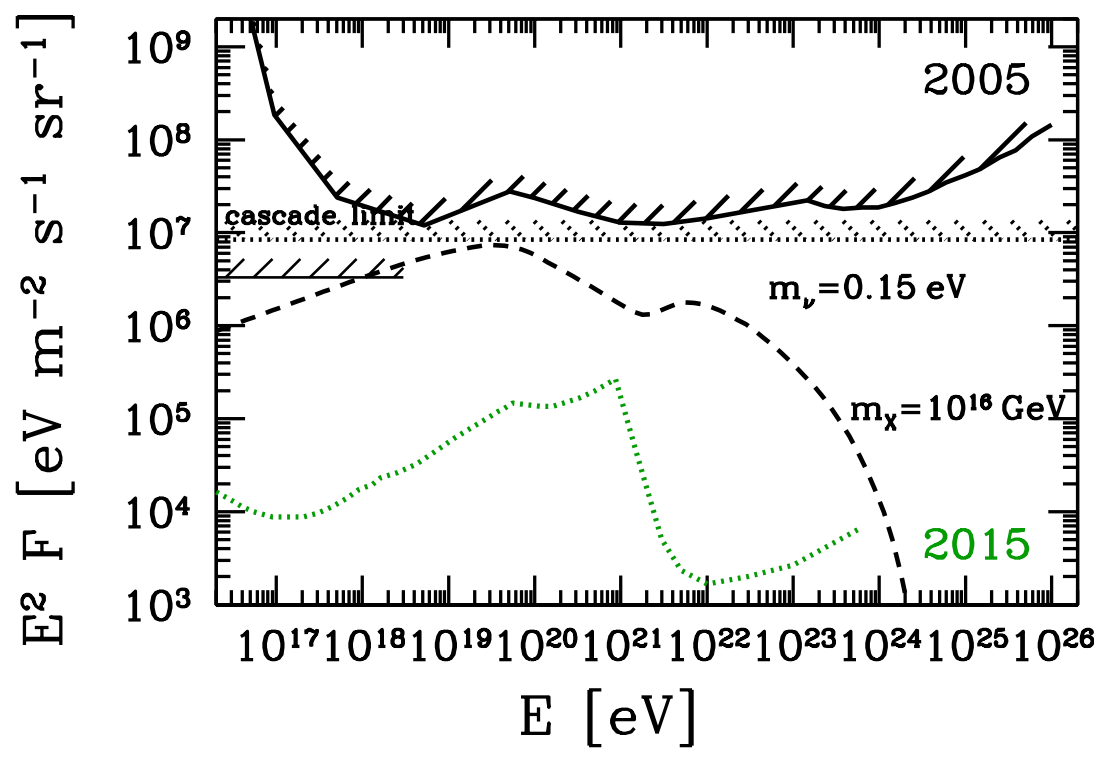

Figure 5. Present (2005) limits on the neutrino flux and projected sensitivity in ten years from now (2015), together with a prediction from topological defects ${ }^{16}\left(m_{X}=\right.$ $\left.10^{16} \mathrm{GeV}, p=0\right)$. The absorption dip arising from resonant annihilation of the EHEC $\nu$ 's with big bang relic neutrinos of mass $m_{\nu}=0.15 \mathrm{eV}$ into $Z$-bosons is clearly visible.

sizeable event rates from topological defects ${ }^{16}$ (cf. Fig. 5). Note, that, for the first time in cosmic particle physics, the GUT energy scale can be directly probed. Clearly, a precise measurement of the neutrino spectrum from topological defects would have a strong impact on particle physics and cosmology. Its mere existence would signal the existence of topological defects as relics from early phase transitions after inflation. The high end of the spectrum directly reveals the mass of the $X$ particles, and its shape entails detailed information on the particle content of the desert, on the Hubble expansion rate, and on the big bang relic neutrino background. Indeed, as illustrated in Fig. 5, the resonant annihilation of the neutrinos from $X$ particle decays with big bang relic neutrinos would leave its imprints as absorption dips in the measured spectrum ${ }^{56}$. Such a measurement would not only shed light on the existence and the spatial distribution ${ }^{57}$ of the cosmic neutrino background, but would also give important information on the neutrino masses ${ }^{58}$, since the dips occur around the resonance energies $E_{\nu_{i}}^{\text {res }}=4 \times 10^{21} \mathrm{eV}\left(1 \mathrm{eV} / m_{\nu_{i}}\right)$. Note, that, along with a prediction of absorption dips, there goes a prediction of emission features ${ }^{59}$ - protons and photons from hadronic $Z$-decay (" $Z$-bursts") - which may appear as 
a CR flux recovery beyond $E_{\mathrm{GZK}}$ and be measured by EUSO, OWL, or LOFAR $^{16}$.

\section{Conclusions}

The future seems bright in extremely high energetic neutrinos. There are

many observatories under construction, whose combined sensitivity ranges from $10^{7}$ to $10^{17} \mathrm{GeV}$, the energy scale of Grand Unification. In the likely case that appreciable event samples are collected in this energy range, we can expect a strong impact on astrophysics, particle physics, and cosmology.

\section{References}

1. M. Ackermann et al. [AMANDA Collab.], Astropart. Phys. 22 (2005) 339.

2. S. Barwick et al. [ANITA Collaboration], these proceedings and to appear.

3. R. Wischnewski et al. [Baikal Collaboration], arXiv:astro-ph/0507698.

4. N. G. Lehtinen, P. W. Gorham, A. R. Jacobson and R. A. Roussel-Dupre, Phys. Rev. D 69 (2004) 013008.

5. P. W. Gorham, C. L. Hebert, K. M. Liewer, C. J. Naudet, D. Saltzberg and D. Williams, Phys. Rev. Lett. 93 (2004) 041101.

6. I. Kravchenko, arXiv:astro-ph/0306408.

7. P. Gorham et al. [ANITA Collaboration], NASA Proposal SMEX03-0004-0019.

8. S. Bottai and S. Giurgola [EUSO Collaboration], in: Proc. 28th International Cosmic Ray Conference, Tsukuba, Japan, 2003, pp. 1113-1116; S. Bottai [EUSO Collaboration], to appear in: Proc. Incontro Nazionale di Astrofisica delle Alte Energie, Roma, 2003.

9. J. Ahrens et al. [IceCube Collab.], Nucl. Phys. Proc. Suppl. 118 (2003) 388.

10. O. Scholten, J. Bacelar, R. Braun, A. G. de Bruyn, H. Falcke, B. Stappers and R. G. Strom, arXiv:astro-ph/0508580.

11. F. W. Stecker, J. F. Krizmanic, L. M. Barbier, E. Loh, J. W. Mitchell, P. Sokolsky and R. E. Streitmatter, Nucl. Phys. Proc. Suppl. 136C (2004) 433; J. F. Krizmanic, private communications.

12. X. Bertou, P. Billoir, O. Deligny, C. Lachaud and A. Letessier-Selvon, Astropart. Phys. 17 (2002) 183.

13. P. Gorham, D. Saltzberg, A. Odian, D. Williams, D. Besson, G. Frichter and S. Tantawi, Nucl. Instrum. Meth. A 490 (2002) 476; private commun.

14. M. Ahlers, L. A. Anchordoqui, H. Goldberg, F. Halzen, A. Ringwald and T. J. Weiler, Phys. Rev. D 72 (2005) 023001.

15. Z. Fodor, S. D. Katz, A. Ringwald and H. Tu, JCAP 0311 (2003) 015.

16. Z. Fodor, S. D. Katz, A. Ringwald, T. J. Weiler and Y. Y. Y. Wong, DESY 05-165.

17. E. Waxman and J. N. Bahcall, Phys. Rev. D 59 (1999) 023002; K. Mannheim, R. J. Protheroe and J. P. Rachen, Phys. Rev. D 63 (2001) 023003.

18. M. Nagano et al., J. Phys. G 18 (1992) 423. 
19. M. Takeda et al. [AGASA Collab.], Phys. Rev. Lett. 81 (1998) 1163; Astropart. Phys. 19 (2003) 447; http://www-akeno.icrr.u-tokyo.ac.jp/AGASA/ 20. D. J. Bird et al. [Fly's Eye Collaboration], Phys. Rev. Lett. 713401 (1993); Astrophys. J. 424, 491 (1994); Astrophys. J. 441, 144 (1995).

21. T. Abu-Zayyad et al. [HiRes Collaboration], Astropart. Phys. 23 (2005) 157.

22. V. Berezinsky, A. Z. Gazizov and S. I. Grigorieva, arXiv:hep-ph/0204357; Phys. Lett. B 612 (2005) 147.

23. Z. Fodor, S. D. Katz, A. Ringwald and H. Tu, Phys. Lett. B 561 (2003) 191.

24. K. Greisen, Phys. Rev. Lett. 16 (1966) 748; G. T. Zatsepin and V. A. Kuzmin, JETP Lett. 4 (1966) 78.

25. D. R. Bergman [HiRes Collab.], Nucl. Phys. Proc. Suppl. 136 (2004) 40.

26. H. Tu, DESY-THESIS-2004-018; unpubl.

27. J. Kwiecinski, A. D. Martin and A. M. Stasto, Phys. Rev. D 59 (1999) 093002.

28. R. Gandhi, C. Quigg, M. H. Reno and I. Sarcevic, Phys. Rev. D 58 (1998) 093009.

29. M. Glück, S. Kretzer and E. Reya, Astropart. Phys. 11 (1999) 327.

30. K. Kutak and J. Kwiecinski, Eur. Phys. J. C 29 (2003) 521.

31. C. Adloff et al. [H1 Collaboration], Eur. Phys. J. C 30 (2003) 1.

32. S. Chekanov et al. [ZEUS Collaboration], Eur. Phys. J. C 32 (2003) 1.

33. H. Aoyama and H. Goldberg, Phys. Lett. B 188 (1987) 506; A. Ringwald, Nucl. Phys. B 330 (1990) 1; O. Espinosa, Nucl. Phys. B 343 (1990) 310.

34. I. Antoniadis, Phys. Lett. B 246 (1990) 377; J. D. Lykken, Phys. Rev. D 54 (1996) 3693; N. Arkani-Hamed, S. Dimopoulos and G. R. Dvali, Phys. Lett. B 429 (1998) 263; L. Randall and R. Sundrum, Phys. Rev. Lett. 83 (1999) 3370 .

35. R. M. Baltrusaitis et al., Phys. Rev. D 31 (1985) 2192.

36. S. Yoshida et al. [AGASA Collaboration], in: Proc. 27th International Cosmic Ray Conference, Hamburg, Germany, 2001, p. 1142

37. V. S. Berezinsky and A. Y. Smirnov, Phys. Lett. B 48 (1974) 269.

38. D. A. Morris and A. Ringwald, Astropart. Phys. 2 (1994) 43.

39. R. J. Protheroe and P. A. Johnson, Astropart. Phys. 4 (1996) 253.

40. L. A. Anchordoqui, Z. Fodor, S. D. Katz, A. Ringwald and H. Tu, JCAP 0506 (2005) 013.

41. V. S. Berezinsky and G. T. Zatsepin, Phys. Lett. B 28 (1969) 423.

42. M. Ahlers, A. Ringwald and H. Tu, arXiv:astro-ph/0506698.

43. A. Ringwald, JHEP 0310 (2003) 008.

44. T. Han and D. Hooper, Phys. Lett. B 582 (2004) 21.

45. L. A. Anchordoqui, J. L. Feng and H. Goldberg, Phys. Lett. B 535 (2002) 302.

46. W. S. Burgett, G. Domokos and S. Kovesi-Domokos, Nucl. Phys. Proc. Suppl. 136 (2004) 327.

47. T. Han and D. Hooper, New J. Phys. 6 (2004) 150.

48. L. Anchordoqui, T. Han, D. Hooper and S. Sarkar, arXiv:hep-ph/0508312.

49. J. Polchinski, arXiv:hep-th/0412244.

50. I. Tkachev, S. Khlebnikov, L. Kofman and A. D. Linde, Phys. Lett. B 440 
(1998) 262.

51. P. Bhattacharjee and G. Sigl, Phys. Rept. 327 (2000) 109.

52. P. Bhattacharjee, C. T. Hill and D. N. Schramm, Phys. Rev. Lett. 69 (1992) 567.

53. R. Aloisio, V. Berezinsky and M. Kachelriess, Phys. Rev. D 69 (2004) 094023.

54. M. Birkel and S. Sarkar, Astropart. Phys. 9 (1998) 297; V. Berezinsky and M. Kachelriess, Phys. Rev. D 63 (2001) 034007.

55. Z. Fodor and S. D. Katz, Phys. Rev. Lett. 86 (2001) 3224; S. Sarkar and R. Toldra, Nucl. Phys. B 621 (2002) 495; C. Barbot and M. Drees, Astropart. Phys. 20 (2003) 5.

56. T. J. Weiler, Phys. Rev. Lett. 49 (1982) 234.

57. S. Singh and C. P. Ma, Phys. Rev. D 67 (2003) 023506; A. Ringwald and Y. Y. Y. Wong, JCAP 0412 (2004) 005.

58. B. Eberle, A. Ringwald, L. Song and T. J. Weiler, Phys. Rev. D 70 (2004) 023007; G. Barenboim, O. Mena Requejo and C. Quigg, Phys. Rev. D 71 (2005) 083002; J. C. D'Olivo, L. Nellen, S. Sahu and V. Van Elewyck, arXiv:astro-ph/0507333.

59. D. Fargion, B. Mele and A. Salis, Astrophys. J. 517 (1999) 725; T. J. Weiler, Astropart. Phys. 11 (1999) 303; Z. Fodor, S. D. Katz and A. Ringwald, Phys. Rev. Lett. 88 (2002) 171101; JHEP 0206 (2002) 046; A. Ringwald, T. J. Weiler and Y. Y. Y. Wong, Phys. Rev. D 72 (2005) 043008. 